УДК 517.53

I. V. Andrusyak, P. V. Filevych

\title{
THE MINIMAL GROWTH OF ENTIRE FUNCTIONS WITH GIVEN ZEROS ALONG UNBOUNDED SETS
}

I. V. Andrusyak, P. V. Filevych. The minimal growth of entire functions with given zeros along unbounded sets, Mat. Stud. 54 (2020), 146-153.

Let $l$ be a continuous function on $\mathbb{R}$ increasing to $+\infty$, and $\varphi$ be a positive function on $\mathbb{R}$. We proved that the condition

$$
\varliminf_{x \rightarrow+\infty} \frac{\varphi(\ln [x])}{\ln x}>0
$$

is necessary and sufficient in order that for any complex sequence $\left(\zeta_{n}\right)$ with $n(r) \geq l(r), r \geq r_{0}$, and every set $E \subset \mathbb{R}$ which is unbounded from above there exists an entire function $f$ having zeros only at the points $\zeta_{n}$ such that

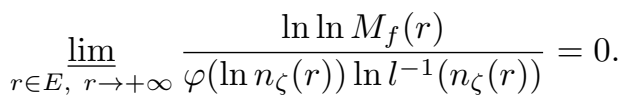

Here $n(r)$ is the counting function of $\left(\zeta_{n}\right)$, and $M_{f}(r)$ is the maximum modulus of $f$.

1. Introduction. Let $\mathcal{Z}$ be the class of all complex sequences

$$
\zeta=\left(\zeta_{n}\right) \text { such that } 0<\left|\zeta_{0}\right| \leq\left|\zeta_{1}\right| \leq \ldots \text { and } \zeta_{n} \rightarrow \infty(n \rightarrow+\infty) .
$$

For every sequence $\zeta \in \mathcal{Z}$, by $\mathcal{E}_{\zeta}$ we denote the class of all entire functions whose sequence of zeros, enumerated (counted with multiplicity) in non-decreasing order of their moduli, coincides with the sequence $\zeta \in \mathcal{Z}$, and let

$$
n_{\zeta}(r)=\sum_{\left|\zeta_{n}\right| \leq r} 1
$$

be the counting function of this sequence.

Suppose that $E \subset \mathbb{R}$ is a measurable set. As usual, the value $\int_{E \cap(1,+\infty)} d \ln r$ is called the logarithmic measure of the set $E$, and the value

$$
\varlimsup_{r \rightarrow+\infty} \frac{1}{\ln r} \int_{E \cap(1,+\infty)} d \ln r
$$

is called the upper logarithmic density of this set.

For an entire function $f$ and every $r \geq 0$ we denote $M_{f}(r)=\max \{|f(z)|:|z|=r\}$.

A. A. Goldberg ([1]) proved the following theorem.

2010 Mathematics Subject Classification: 30D15, 30D20.

Keywords: entire function; maximum modulus; zeros; counting function. doi:10.30970/ms.54.2.146-153

(C) I. V. Andrusyak, P. V. Filevych, 2020 
Theorem A. Let $\delta>2$. For any sequence $\zeta \in \mathcal{Z}$ satisfying the condition

$$
\varliminf_{r \rightarrow+\infty} \frac{\ln n_{\zeta}(r)}{\ln r}>0
$$

there exist an entire function $f \in \mathcal{E}_{\zeta}$ and a set $E$ of finite logarithmic measure such that outside the set $E$ one has

$$
\ln \ln M_{f}(r)=o\left(\ln ^{\delta} n_{\zeta}(r)\right), \quad r \rightarrow+\infty
$$

In addition, A. A. Goldberg ([1]) showed that Theorem A is not valid in the case $\delta=$ 1 , and also posed the question of whether in this theorem the condition $\delta>2$ can be replaced by the one $\delta>1$. The negative answer to Goldberg's question was obtained by W. Bergweiler ([2]), who showed that Theorem A is not true anymore even in the case $\delta=2$.

Theorem B $([2])$. Let $\alpha \in(0,+\infty)$. There exists a sequence $\zeta \in \mathcal{Z}$ satisfying the condition

$$
\varliminf_{r \rightarrow+\infty} \frac{\ln n_{\zeta}(r)}{\ln r}=\alpha
$$

such that for any entire function $f \in \mathcal{E}_{\zeta}$ along some set $E_{f}$ of infinite logarithmic measure one has

$$
\ln ^{2} n_{\zeta}(r)=o\left(\ln \ln M_{f}(r)\right), \quad r \rightarrow+\infty .
$$

If we require the validity of relation (2) not outside a small set such as a set of finite logarithmic measure, but only along some increasing to $+\infty$ sequence of values $r$, this relation can also be valid in the case of $\delta=2$.

Theorem $\mathbf{C}([2])$. For any sequence $\zeta \in \mathcal{Z}$ that satisfies condition (1) and every unbounded from above set $E \subset \mathbb{R}$ there exists a function $f \in \mathcal{E}_{\zeta}$ such that

$$
\varliminf_{r \in E, r \rightarrow+\infty} \frac{\ln \ln M_{f}(r)}{\ln ^{2} n_{\zeta}(r)}=0
$$

The following statement shows that under conditions of Theorem C relation (4) is final in some sense.

Theorem D ([2]). Suppose $\alpha \in(0,+\infty)$ and $\phi$ is a function decreasing to 0 on $\mathbb{R}$. Then there exist a sequence $\zeta \in \mathcal{Z}$ satisfying condition (3) and a set $E \subset \mathbb{R}$ of upper logarithmic density 1 such that for any function $f \in \mathcal{E}_{\zeta}$ we have

$$
\lim _{r \in E, r \rightarrow+\infty} \frac{\ln \ln M_{f}(r)}{\ln ^{2} n_{\zeta}(r) \phi\left(\ln n_{\zeta}(r)\right)}=+\infty .
$$

Remark 1. W. Bergweiler [2] actually proved to some extent deeper results than Theorems A and B. Particularly, from results obtained in [2] it follows that a function $f \in \mathcal{E}_{\zeta}$ and an exceptional set $E$ in Theorem A can be selected independently of the number $\delta>2$. On the other hand, Theorem $\mathrm{C}$ shows that the set $E_{f}$ in Theorem $\mathrm{B}$ is dependends on $f$. 
Theorems A and B describe the minimal growth of an entire function having given sequence of zeros $\zeta$ only in the case when the growth of the counting function $n_{\zeta}(r)$ for this sequence is bounded from below by some power function $r^{\alpha}$. Analogs of Theorems A and $\mathrm{B}$ were obtained in [3] in the case when the growth of the function $n_{\zeta}(r)$ is bounded from below by a function of the form $\ln ^{\alpha} r$. Theorems $A$ and $B$ were extended in [4] to the case when the restrictions mentioned above are of any possible kind.

By $L$ we denote the class of all continuous on $\mathbb{R}$ functions increasing to $+\infty$.

Theorem $\mathbf{E}([4])$. Let $l \in L$. For any sequence $\zeta \in \mathcal{Z}$ satisfying the condition

$$
n_{\zeta}(r) \geq l(r) \quad\left(r \geq r_{0}\right),
$$

there exist an entire function $f \in \mathcal{E}_{\zeta}$ and a set $E \subset \mathbb{R}$ of finite logarithmic measure such that for every $\delta>1$ outside the set $E$ one has

$$
\ln \ln M_{f}(r)=o\left(\ln ^{\delta} n_{\zeta}(r) \ln l^{-1}\left(n_{\zeta}(r)\right)\right), \quad r \rightarrow+\infty .
$$

Theorem $\mathbf{F}([4])$. Let $l \in L$. There exists a sequence $\zeta \in \mathcal{Z}$ that satisfies condition (5) such that $n_{\zeta}(r-0)=l(r)$ on an unbounded from above set of values $r$ and for any entire function $f \in \mathcal{E}_{\zeta}$ along some set $E_{f}$ of infinite logarithmic measure one has

$$
\ln n_{\zeta}(r) \ln l^{-1}\left(n_{\zeta}(r)\right)=o\left(\ln \ln M_{f}(r)\right), \quad r \rightarrow+\infty .
$$

The goal of our paper is generalizations of Theorems $\mathrm{C}$ and $\mathrm{D}$ for the case of any possible lower bound on the growth of the counting function $n_{\zeta}(r)$ for a sequence $\zeta \in \mathcal{Z}$.

Theorem 1. Let $l \in L$. Then for any sequence $\zeta \in \mathcal{Z}$ that satisfies condition (5), and for every unbounded from above set $E \subset \mathbb{R}$ there exists a function $f \in \mathcal{E}_{\zeta}$ for which

$$
\varliminf_{r \in E, r \rightarrow+\infty} \frac{\ln \ln M_{f}(r)}{\ln n_{\zeta}(r) \ln l^{-1}\left(n_{\zeta}(r)\right)}=0 .
$$

Theorem 2. Let $l \in L$ and $\varphi$ be a function which is positive on $\mathbb{R}$ and such that

$$
\varliminf_{x \rightarrow+\infty} \frac{\varphi(\ln [x])}{\ln x}=0 .
$$

Then there exists a sequence $\zeta \in \mathcal{Z}$ such that condition (5) holds and $n_{\zeta}(r-0)=l(r)$ on an unbounded from above set of values $r$, and also there exists a set $E \subset \mathbb{R}$ of upper logarithmic density 1 such that for any function $f \in \mathcal{E}_{\zeta}$ one has

$$
\lim _{r \in E, r \rightarrow+\infty} \frac{\ln \ln M_{f}(r)}{\varphi\left(\ln n_{\zeta}(r)\right) \ln l^{-1}\left(n_{\zeta}(r)\right)}=+\infty .
$$

Immediately from Theorems 1 and 2 we obtain the following theorem.

Theorem 3. Let $l \in L$ and $\varphi$ be a function which is positive on $\mathbb{R}$. Then the condition

$$
\varliminf_{x \rightarrow+\infty} \frac{\varphi(\ln [x])}{\ln x}>0
$$

is necessary and sufficient in order that for any sequence $\zeta \in \mathcal{Z}$ that satisfies condition (5) and for every unbounded from above set $E \subset \mathbb{R}$ there exists an entire function $f \in \mathcal{E}_{\zeta}$ such that

$$
\varliminf_{r \in E, r \rightarrow+\infty} \frac{\ln \ln M_{f}(r)}{\varphi\left(\ln n_{\zeta}(r)\right) \ln l^{-1}\left(n_{\zeta}(r)\right)}=0
$$


To prove Theorems 1 and 2, we need some auxiliary results which are formulated in the next section.

We have noted that the paper [1] also inspired other problems related to the description of the minimum growth of entire functions with given zeros. In particular, some of these problems were solved in papers $[5,6,7,8,9]$.

\section{Auxiliary results.}

Lemma 1 ([4]). For every sequence $\zeta \in \mathcal{Z}$ there exists a nonnegative sequence $\left(\lambda_{n}\right)$ having the following properties:

(i) $\lambda_{n} \sim \ln n / \ln \left|\zeta_{n}\right|$, as $n \rightarrow \infty$;

(ii) for any sequence of nonnegative integers $\left(p_{n}\right)$ such that $p_{n} \geq\left[\lambda_{n}\right], n \geq n_{0}$, the product

$$
f(z)=\prod_{n=0}^{\infty} E\left(\frac{z}{\zeta_{n}}, p_{n}\right)
$$

defines an entire function $f \in \mathcal{E}(\zeta)$, moreover,

$$
\ln M_{f}(r) \leq G_{f}(r):=\sum_{n=0}^{\infty}\left(\frac{r}{\left|\zeta_{n}\right|}\right)^{p_{n}+1} .
$$

Let $f$ be an entire function, $r>0$, and $c_{p}(r)$ be the $p$-th Fourier coefficient of the function $\ln \left|f\left(r e^{i \theta}\right)\right|$, that is,

$$
c_{p}(r)=\frac{1}{2 \pi} \int_{0}^{2 \pi} e^{-i p \theta} \ln \left|f\left(r e^{i \theta}\right)\right| d \theta, \quad p \in \mathbb{Z} .
$$

Suppose that $f(0) \neq 0$ and

$$
\ln f(z)=\sum_{p=0}^{\infty} a_{p} z^{p}
$$

near the point $z=0$. Then, applying the Poisson-Jensen formula (see [10, p. 16-17]), for every integer $p \geq 1$ we have

$$
c_{p}(r)=\frac{1}{2} a_{p} r^{p}+\frac{1}{2 p} \sum_{\left|\zeta_{n}\right|<r}\left(\left(\frac{r}{\zeta_{n}}\right)^{p}-\left(\overline{\frac{\zeta_{n}}{r}}\right)^{p}\right),
$$

where $\zeta_{n}$ are zeros of the function $f$. Moreover, the following lemma is valid.

Lemma 2 ([2]). For any entire function $f$ and every integer $n \geq 1$ the inequality

$$
\left|c_{n}(r)\right| \leq \ln M_{f}(r) \quad(r>0)
$$

is satisfied. 


\section{Proof of Theorems.}

Proof of Theorem 1. Suppose that $\zeta \in \mathcal{Z}$ is a fixed sequence such that $n_{\zeta}(r) \geq l(r), r \geq r_{0}$, and $\lambda=\left(\lambda_{n}\right)$ is a sequence whose existence for given $\zeta$ is asserted by Lemma 1 . Then there exists a non-decreasing sequence of non-negative integers $\left(q_{n}\right)$ such that $q_{n} \geq\left[\lambda_{n}\right]$ for $n \geq n_{0}$, and also $q_{n} \rightarrow+\infty$ and $q_{n}=o(\ln n)$ as $n \rightarrow \infty$.

Let us consider the series

$$
\sum_{n=0}^{\infty}\left(\frac{r}{\left|\zeta_{n}\right|}\right)^{q_{n}+1}
$$

which is convergent for all $r \geq 0$, and for every $r \geq 0$ we put $n(r)=n_{\zeta}(r)$. We also put

$$
m(r)=\min \left\{k \geq n(r)+2: \sum_{n=k}^{\infty}\left(\frac{r}{\left|\zeta_{n}\right|}\right)^{q_{n}+1} \leq 1\right\}, \quad \gamma(r)=\frac{\ln (m(r)-n(r))}{\ln \left|\zeta_{n(r)+1}\right|-\ln r} .
$$

Let $E \subset \mathbb{R}$ be a set unbounded from above. In this set we choose a sequence $\left(r_{k}\right)$ increasing to $+\infty$ such that for every integer $k \geq 0$ the following inequality is valid:

$$
n\left(r_{k+1}\right)>m\left(r_{k}\right), \quad q_{n\left(r_{k+1}\right)} \geq \gamma\left(r_{k}\right)+1 .
$$

For any integer $t \geq 0$ let us denote $p_{t}=\max \left\{\left[\gamma\left(r_{k}\right)\right]+1, q_{t}\right\}$ if $t \in\left[n\left(r_{k}\right)+1, m\left(r_{k}\right)\right)$ for some $k \geq 0$, and put $p_{t}=q_{t}$ if $t \notin H$, where

$$
H=\bigcup_{k=0}^{\infty}\left[n\left(r_{k}\right)+1, m\left(r_{k}\right)\right)
$$

Note that $n\left(r_{k}\right) \notin H$ for every $k \geq 0$.

Let us prove that $p_{t} \leq q_{n\left(r_{k}\right)}$ for all $t \leq n\left(r_{k}\right)$ and $k \geq 0$. If $t \notin H$, we have $p_{t}=q_{t} \leq q_{n\left(r_{k}\right)}$, because the sequence $\left(q_{n}\right)$ is non-decreasing. But if $t \in H$, then we have $t \in\left[n\left(r_{j}\right)+1, m\left(r_{j}\right)\right)$ for some $j<k$, and therefore either

$$
p_{t}=\left[\gamma\left(r_{j}\right)\right]+1 \leq \gamma\left(r_{j}\right)+1 \leq q_{n\left(r_{j+1}\right)} \leq q_{n\left(r_{k}\right)},
$$

or $p_{t}=q_{t} \leq q_{n\left(r_{k}\right)}$ again.

It is also clear that $p_{t} \geq q_{t}$ for all $t \geq 0$. Therefore, by Lemma 1 , product (9) defines an entire function $f \in \mathcal{E}_{\zeta}$ for which inequality (10) is true. For each $k \geq 0$, we successively have

$$
\begin{gathered}
\sum_{t \leq n\left(r_{k}\right)}\left(\frac{r_{k}}{\left|\zeta_{t}\right|}\right)^{p_{t}+1} \leq r_{k}^{q_{n\left(r_{k}\right)}+1} \sum_{t \leq n\left(r_{k}\right)}\left(\frac{1}{\left|\zeta_{t}\right|}\right)^{p_{t}+1} \leq r_{k}^{q_{n\left(r_{k}\right)}+1} G(1), \\
\sum_{n\left(r_{k}\right)<t<m\left(r_{k}\right)}\left(\frac{r_{k}}{\left|\zeta_{t}\right|}\right)^{p_{t}+1} \leq\left(m\left(r_{k}\right)-n\left(r_{k}\right)\right)\left(\frac{r_{k}}{\left|\zeta_{n\left(r_{k}\right)+1}\right|}\right)^{\gamma\left(r_{k}\right)}=1, \\
\sum_{t \geq m\left(r_{k}\right)}\left(\frac{r_{k}}{\left|\zeta_{t}\right|}\right)^{p_{t}+1} \leq \sum_{t \geq m\left(r_{k}\right)}\left(\frac{r_{k}}{\left|\zeta_{t}\right|}\right)^{q_{t}+1} \leq 1 .
\end{gathered}
$$

Thus, applying inequality (10), we obtain

$$
\ln \ln M_{f}\left(r_{k}\right) \leq(1+o(1)) q_{n\left(r_{k}\right)} \ln r_{k}=o\left(\ln n\left(r_{k}\right) \ln l^{-1}\left(n\left(r_{k}\right)\right)\right), \quad k \rightarrow \infty .
$$

It implies (6). 
Proof of Theorem 2. Without loss of generality, we may suppose that $l(1)<0$.

Let $\left(\delta_{k}\right)$ be any decreasing to 0 sequence of points in the interval $(0,1)$. From condition

(7) it follows the existence of an increasing sequence of integers $\left(n_{k}\right)$ such that $n_{0}=0$ and

$$
\begin{aligned}
2 r_{k} & \leq r_{k+1}^{\delta_{k}}, \quad \ln m_{k} \\
\ln m_{k} & \geq 2 k \ln r_{k}, \quad \varepsilon_{k} \leq \delta_{k}, \quad \varepsilon_{k} \ln r_{k} \leq 1,
\end{aligned}
$$

for every $k \geq 0$, where

$$
\varepsilon_{k}=\left(\frac{\varphi\left(\ln n_{k+1}\right)}{\ln n_{k+1}}\right)^{1 / 3}, \quad r_{k}=l^{-1}\left(n_{k}\right), \quad m_{k}=n_{k+1}-n_{k} \quad(k \geq 0) .
$$

Note that $r_{0}>1$ by inequality $l(1)<0$.

For any $k \geq 0$ we also denote

$$
p_{k}=\left[\frac{\ln m_{k}}{2 \ln r_{k}}\right]+1
$$

Applying the first inequality in (14), we see that $p_{k} \rightarrow+\infty, k \rightarrow \infty$. Moreover,

$$
\ln \frac{m_{k}}{4 p_{k} r_{k}^{p_{k}}}=\ln m_{k}-p_{k} \ln r_{k}-\ln p_{k}-\ln 4=\left(\frac{1}{2}+o(1)\right) \ln m_{k} \rightarrow+\infty, \quad k \rightarrow \infty .
$$

Construct the sequence $\zeta$ as following

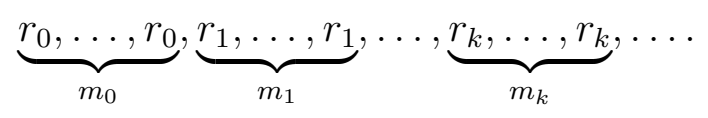

If $r \in\left[0, r_{0}\right)$, then $n_{\zeta}(r)=0=l\left(r_{0}\right)>l(r)$. But if $r \in\left[r_{k}, r_{k+1}\right)$ for some $k \geq 0$, then we get

$$
n_{\zeta}(r)=\sum_{j=0}^{k} m_{j}=n_{k+1}=l\left(r_{k+1}\right)>l(r)
$$

Therefore, $n_{\zeta}(r)>l(r)$ for every $r \geq 0$. Moreover, $n_{\zeta}\left(r_{k}-0\right)=l\left(r_{k}\right)$ for all $k \geq 0$.

Denote $s_{k}=r_{k+1}^{\delta_{k}}, k \geq 0$. Then, applying the second inequality in (13), we see that $r_{k}<s_{k}<r_{k+1}$ for every $k \geq 0$. Let $E=\cup_{k=0}^{\infty}\left(s_{k}, r_{k+1}\right)$. For the set $E$ we have

$$
\varlimsup_{r \rightarrow+\infty} \frac{1}{\ln r} \int_{E \cap(1, r)} \frac{d t}{t} \geq \varlimsup_{k \rightarrow \infty} \frac{1}{\ln r_{k+1}} \int_{s_{k}}^{r_{k+1}} \frac{d t}{t}=\varlimsup_{k \rightarrow \infty}\left(1-\delta_{k}\right)=1 .
$$

Thus, $E$ is a set of upper logarithmic density 1 .

We need to prove that for any function $f \in \mathcal{E}_{\zeta}$ relation (8) is true.

Let $f \in \mathcal{E}_{\zeta}$. Then in the disc $\left\{z \in \mathbb{C}:|z|<r_{0}\right\}$ the function $f$ has not zeros. Since $r_{0}>1$, we deduce that if we have (11) near the point $z=0$, then the sequence $\left(a_{p}\right)$ is bounded, that is, $C:=\sup \left\{\left|a_{p}\right|: p \in \mathbb{N}\right\}<+\infty$.

Suppose $c_{p}(r)$ is the $p$-th Fourier coefficient of the function $\ln \left|f\left(r e^{i \theta}\right)\right|$. Using equality (12) and the first inequality in (13), which can be rewritten as $2 r_{k} \leq s_{k}$, and, taking into 
account that the sequence $\zeta$ is positive for all $k \geq 0$ and $p \geq 1$, we obtain

$$
\begin{aligned}
&\left|c_{p}\left(s_{k}\right)\right| \geq \frac{1}{2 p} \sum_{\zeta_{n}<s_{k}}\left(\left(\frac{s_{k}}{\zeta_{n}}\right)^{p}-\left(\frac{\zeta_{n}}{s_{k}}\right)^{p}\right)-C s_{k}^{p} \geq \frac{1}{2 p} \sum_{r_{k} \leq \zeta_{n}<s_{k}}\left(\left(\frac{s_{k}}{\zeta_{n}}\right)^{p}-\left(\frac{\zeta_{n}}{s_{k}}\right)^{p}\right)-C s_{k}^{p}= \\
&= \frac{m_{k}}{2 p}\left(\left(\frac{s_{k}}{r_{k}}\right)^{p}-\left(\frac{r_{k}}{s_{k}}\right)^{p}\right)-C s_{k}^{p}=\frac{m_{k}}{2 p}\left(\frac{s_{k}}{r_{k}}\right)^{p}\left(1-\left(\frac{r_{k}}{s_{k}}\right)^{2 p}\right)-C s_{k}^{p} \geq \\
& \geq \frac{m_{k}}{4 p}\left(\frac{s_{k}}{r_{k}}\right)^{p}-C s_{k}^{p} \geq s_{k}^{p}\left(\frac{m_{k}}{4 p r_{k}^{p}}-C\right) .
\end{aligned}
$$

Further, from (15), it follows that $\left|c_{p_{k}}\left(s_{k}\right)\right| \geq s_{k}^{p_{k}}, k \geq k_{0}$.

Using this inequality together with (13) and (14), for all $r \in\left(s_{k}, r_{k+1}\right)$ and $k \geq k_{0}$ we obtain

$$
\begin{gathered}
\ln \ln M_{f}(r) \geq \ln \ln M_{f}\left(s_{k}\right) \geq \ln \left|c_{p}\left(s_{k}\right)\right| \geq p_{k} \ln s_{k} \geq \\
\geq \frac{\ln m_{k}}{2 \ln r_{k}} \delta_{k} \ln r_{k+1} \geq \frac{1-\delta_{k}}{2} \ln n_{k+1} \frac{1}{\ln r_{k}} \delta_{k} \ln l^{-1}\left(n_{k+1}\right) \geq \\
\geq \frac{1-\delta_{k}}{2} \frac{\varphi\left(\ln n_{k+1}\right)}{\varepsilon_{k}^{3}} \varepsilon_{k} \varepsilon_{k} \ln l^{-1}\left(n_{k+1}\right)=\frac{1-\delta_{k}}{2 \varepsilon_{k}} \varphi\left(\ln n_{\zeta}(r)\right) \ln l^{-1}\left(n_{\zeta}(r)\right) .
\end{gathered}
$$

Hence, we get (8).

Remark 2. To characterize the growth of an entire function $f$, we can use besides $\ln M_{f}(r)$ its Nevanlinna characteristic function

$$
T_{f}(r)=\frac{1}{2 \pi} \int_{0}^{2 \pi} \ln ^{+}\left|f\left(r e^{i \theta}\right)\right| d \theta, \quad r \geq 0 .
$$

We note that in Theorems 1, 2, and 3 the function $\ln M_{f}(r)$ can be replaced by the function $T_{f}(r)$. The validity of this replacement in relation (6) follows from the inequality $T_{f}(r) \leq$ $\ln ^{+} M_{f}(r)$. To justify the possibility of replacing $\ln M_{f}(r)$ by $T_{f}(r)$ in relation (8), it is sufficient to repeat the proof of Theorem 2 using the inequality $\left|c_{n}(r)\right| \leq 2 T_{f}(r)$ (see, for example, [10, p. 340]) instead of the inequality $\left|c_{n}(r)\right| \leq \ln M_{f}(r)$ from Lemma 2.

\section{REFERENCES}

1. A.A. Gol'dberg, The representation of a meromorphic function in the form of a quotient of entire functions, Izv. Vyssh. Uchebn. Zaved. Mat., 10 (1972), 13-17. (in Russian)

2. W. Bergweiler, A question of Gol'dberg concerning entire functions with prescribed zeros, J. Anal. Math., 63 (1994), 121-129.

3. I.V. Khyrivskyi, Minimal growth of entire function with given sequence of zeros, Mat. Stud., 3 (1994), 49-52. (in Ukrainian)

4. I.V. Andrusyak, P.V. Filevych, The minimal growth of entire function with given zeros, Nauk. Visn. Chernivets'kogo Univ. Mat., 421 (2008), 13-19. (in Ukrainian)

5. W. Bergweiler, Canonical products of infinite order, J. Reine Angew. Math., 430 (1992), 85-107.

6. J. Miles, On the growth of entire functions with zero sets having infinite exponent of convergence, Ann. Acad. Sci. Fenn. Math., 27 (2002), 69-90. 
7. M.M. Sheremeta, A remark to the construction of canonical products of minimal growth, Mat. Fiz. Anal. Geom., 11 (2004), №2, 243-248.

8. I.V. Andrusyak, P.V. Filevych, The growth of an entire function with a given sequence of zeros, Mat. Stud., 30 (2008), №2, 115-124.

9. I.V. Andrusyak, P.V. Filevych, The growth of entire function with zero sets having integer-valued exponent of convergence, Mat. Stud., 32 (2009), №1, 12-20. (in Ukrainian)

10. A.A. Gol'dberg, I.V. Ostrovskii, Value distribution of meromorphic functions, Moscow: Nauka, 1970. (in Russian)

Department of Mathematics

Lviv Polytechnic National University, Lviv, Ukraine

andrusyak.ivanna@gmail.com

Department of Computational Mathematics and Programming

Lviv Polytechnic National University, Lviv, Ukraine

p.v.filevych@gmail.com 